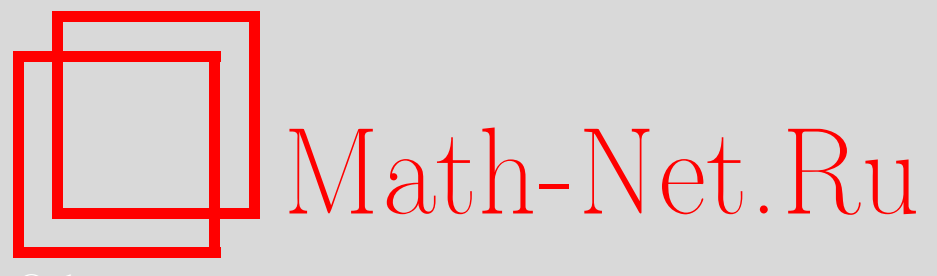

С. А. Бубнов, И. И. Овчинников, А. А. Бубнов, Исследование разрушения и кинетики обезуглероживания толстостенной трубы в условиях водородной коррозии, Вестн. Сам. гос. техн. ун-та. Сер. Физ.-мат. науки, 2012, выпуск 2(), 178-182

DOI: https://doi.org/10.14498/vsgtu960

Использование Общероссийского математического портала Math-Net.Ru подразумевает, что вы прочитали и согласны с пользовательским соглашением

http://www . mathnet.ru/rus/agreement

Параметры загрузки:

IP : 54.224 .60 .19

26 апреля 2023 г., 16:13:10

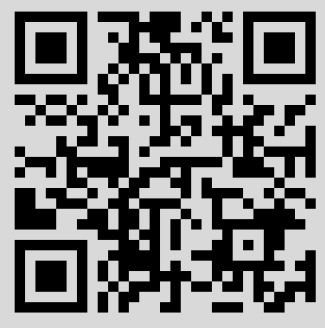




\title{
ИССЛЕДОВАНИЕ РАЗРУШЕНИЯ И КИНЕТИКИ ОБЕЗУГЛЕРОЖИВАНИЯ ТОЛСТОСТЕННОЙ ТРУБЫ В УСЛОВИЯХ ВОДОРОДНОЙ КОРРОЗИИ
}

\author{
С. А. Бубнов ${ }^{1}$, И. И. Овчинников ${ }^{2}$, А.А. Бубнов \\ 1 Балашовский институт (филиал) \\ Саратовского государственного университета им. Н. Г. Чернышевского, \\ 412300, Россия, Саратовская обл., Балашов, ул. Карла Маркса, 29. \\ 2 Саратовский государственный технический университет им. Ю. А. Гагарина, \\ 410054, Россия, Саратов, ул. Политехническая, 77. \\ 3 Рязанский государственный радиотехнический университет, \\ 390005, Россия, Рязань, ул. Гагарина, 59/1. \\ E-mails: serbubnov@rambler.ru, bridgesar@sstu.ru
}

\begin{abstract}
Изучается поведение толстостенного трубопровода в условиях водородной коррозии. Рассматривается моделъный пример толстостенной трубъ из стали 20 с участком локального прогрева. В качестве факторов нагружения в модельном примере использовались внутреннее и наружное давления водородсодержсащей средь. Отмечается, что наибольшее влияние на время до разрушения оказывает величина внутреннего давления водородсодержащей средъ. Также отмечается, что в области повышенных температур процесс обезуглероживания протекает бъстирее.
\end{abstract}

Ключевые слова: водородная коррозия, разрушение, фронт обезуглероживания, толстостенный трубопровод.

Известно, что при высоких температурах (порядка $500 \mathrm{~K}$ ) и относительно высоких давлениях (15-40 МПа) элементы конструкций, контактирующие с водородсодержащей средой, подвергаются воздействию водородной коррозии [1]. Считается, что водород по механизму диффузии проникает в металлы и взаимодействует с углеродом, в результате чего образуется метан. Эта реакция начинается с места контакта водорода с металлом и распространяется по объёму конструктивного элемента, приводя к обезуглероживанию и образованию микротрещин. Свойства материала претерпевают изменения в соответствии со значением параметра химического взаимодействия $\mu$ в данный момент времени в конкретной точке объёма конструкции. В результате разрушение конструктивного элемента наступает раньше, в отличие от его эксплуатации при тех же температурах и давлениях, но без воздействия водородсодержащей среды. Поведение параметра $\mu$ подчиняется уравнению химического взаимодействия:

$$
d \mu / d t=k \mu(1-\mu),
$$

в котором $k=k(P, T, \Pi)$ - коэффициент, зависящий от давления $P$, температуры $T$ и параметра повреждённости $\Pi$. С течением времени параметр $\mu$ изменяется от начального значения $\mu_{0}$ до критического значения $\mu_{\text {кр }}$, проходя через пороговое значение $\mu_{\text {п }}$, наступление которого свидетельствует об окончании инкубационного периода и начале активных химических превращений. Для различных сталей, а

Сергей Алексеевич Бубнов, ассистент, каф. прикладной информатики.

Илья Игоревич Овчинников (к.т.н., доц.), доцент, каф. транспортного строительства.

Алексей Алексеевич Бубнов (к.ф.-м.н., доц.), доцент, каф. вычислительной и прикладной математики. 
также при различных давлениях и температурах длительность инкубационного периода $\tau_{0}$ имеет свое значение. Для стали 20 установлена [2] следующая эмпирическая зависимость $\tau_{0}$ :

$$
\tau_{0}=7,95 \cdot 10^{-4} P^{-1,73} \exp (13500 / T) .
$$

При неоднородном распределении температуры по объёму конструктивного элемента в различных его точках будут происходить химические реакции с разной интенсивностью, а в некоторых точках ещё будет продолжаться инкубационный период. При наличии локального прогрева распределение тепла будет иметь более сложный вид, определить который можно, решив уравнение теплопроводности с соответствующими граничными условиями, например, методом конечных элементов.

Распределение давления по сечению также может носить неоднородный характер. Применительно к толстостенной трубе, находящейся под внутренним $P_{\mathrm{B}}$ и наружным $P_{\mathrm{H}}$ давлениями, распределение давления водорода будет зависеть от соотношения величин $P_{\mathrm{B}}$ и $P_{\mathrm{H}}$. Если $P_{\mathrm{B}}=P_{\mathrm{H}}$, то распределение водорода по сечению будет равномерным и изменение параметра $\mu$ по координатам будет определяться температурой в данной точке в данный момент времени. При действии только внутреннего давления (т. е. $P_{\mathrm{H}}=0$ ) давление водорода будет убывать (от $P=P_{\mathrm{B}}$ до $P=0)$ по экспоненциальной зависимости от внутреннего радиуса трубы $R_{1}$ к наружному $R_{2}$.

В реальных условиях при возникновении аварийных ситуаций температура на внешней поверхности, вероятнее всего, будет изменяться со временем, что приведёт к нестационарности теплового поля. А это, в свою очередь, приведёт к перераспределению параметра $\mu$ на каждом временном шаге. Становится обязательным учёт зависимости модуля Юнга и коэффициента Пуассона от температуры. Будем считать, что вид зависимости не меняется в процессе обезуглероживания материала.

Время до разрушения можно определить, используя уравнение накопления повреждений:

$$
\frac{d \Pi}{d t}=a\left(\frac{\sigma_{\ni}}{1-\Pi}\right)^{b} .
$$

Здесь $a=a(\mu, T)$ и $b=b(\mu, T)$. В качестве эквивалентного напряжения $\sigma_{\ni}$ можно взять [3] интенсивность напряжений. Момент времени, при котором параметр повреждённости $\Pi$ достигнет значения, равного единице, и будет являться временем разрушения конструкции. В зависимости от различного сочетания давлений $P_{\mathrm{B}}$ и $P_{\mathrm{H}}$, распределения теплового поля и от того, какой процент от $P_{\mathrm{B}}$ и $P_{\mathrm{H}}$ составляет именно давление водорода, время до разрушения будет принимать различные значения. Для исследования кинетики обезуглероживания будем придавать значения факторам, руководствуясь интуитивными соображениями.

Рассмотрим модельный пример толстостенной трубы внутреннего радиуса $R_{1}=$ $=5,5$ мм и внешнего радиуса $R_{2}=8$ мм из стали 20 . Не вдаваясь в детали конечноэлементного моделирования [4-7], приведём лишь результаты проведённых исследований. В качестве факторов нагружения использовались следующие параметры: внутреннее $P_{\text {в }}$ и наружное $P_{\mathrm{H}}$ давления водородсодержащей среды при различных температурах $T_{1}$ и $T_{2}$ на внутренней и внешней поверхностях соответственно. Время до разрушения является функцией от указанных факторов. В случае одностороннего воздействия водородсодержащей среды $\left(P_{\mathrm{B}}=19 \mathrm{MПа}\right)$ и при равномерном распределении температуры по объёму $\left(T_{1}=T_{2}=750 \mathrm{~K}\right)$ разрушение по результатам моделирования произойдёт через 480 часов. При этом на момент разрушения сечение трубы обезуглеродится на $67 \%$. Анализ результатов моделирования показывает, что наибольшее перераспределение претерпевают окружные и осевые напряжения (рис. 1, a, б). Параметр повреждённости $\Pi$ достигает своего предельного значения на внутренней поверхности трубы.

Получены зависимости времени до разрушения от внутреннего давления $P_{\mathrm{B}}$ при постоянных температурах. Одна из них $(T=750 \mathrm{~K})$ в качестве примера приведена на рис. 2 , а. Также получены зависимости времени разрушения от температуры при 
постоянных значениях внутреннего давления. На рис. 2, б приведена эта зависимость для $P_{\mathrm{B}}=25$ МПа. Проведённый анализ позволяет сделать вывод, что наибольшее влияние на время до разрушения оказывает величина внутреннего давления.

Анализ поведения трубы при наличии локального прогрева на наружной поверхности показал, что на момент разрушения при $P_{\mathrm{B}}=24$ МПа и изменении температуры локального участка от 720 до $780 \mathrm{~K}$ обезуглероженность сечения в «холодной» зоне составляет $20 \%$, а в локальной зоне - $26 \%$. Авторы объясняют это тем, что в области повышенных температур химические превращения идут более интенсивно и, следовательно, процесс обезуглероживания протекает быстрее. По результатам моделирования разрушение при таких факторах нагружения наступит через 48 часов. Зависимости окружных (а) и осевых (б) напряжений от радиуса в локальнойпрогретой и «холодной» зонах на момент разрушения конструктивного элемента
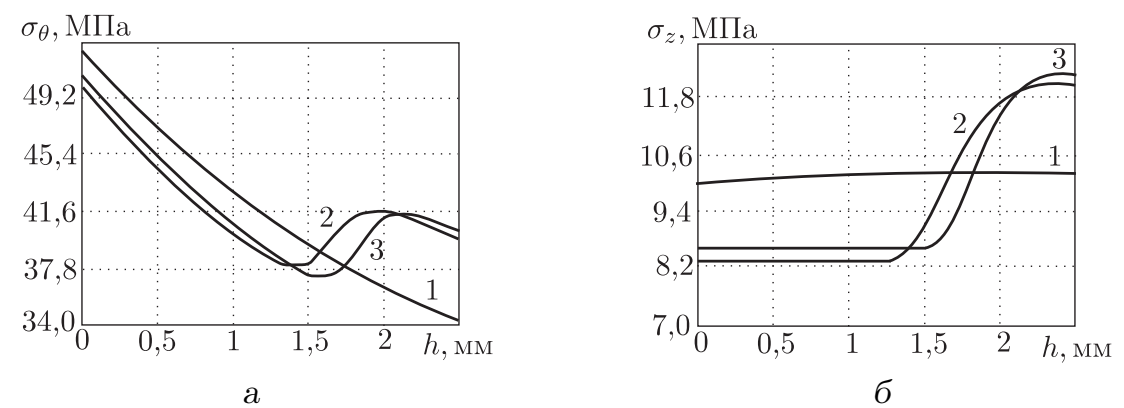

Рис. 1. Распределение окружных (а) и осевых (б) напряжений по толщине трубы $h=r-R_{1}$ в различные моменты времени: $1-t=1$ час; $2-t=200$ час; $3-t=480$ час

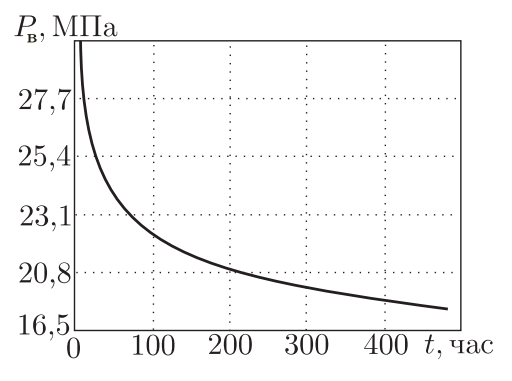

a

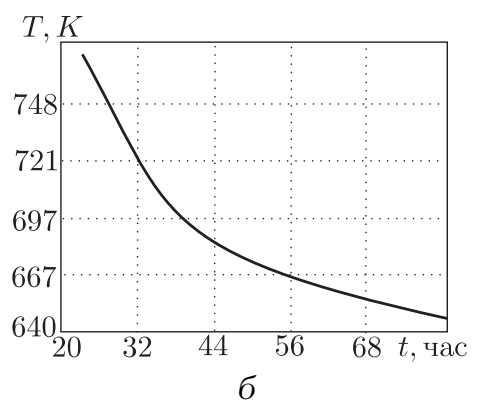

б

Рис. 2. Длительная прочность трубопровода при фиксированной температуре $T=750 \mathrm{~K}($ а) и фиксированном давлении $P=25 \mathrm{MПа} \mathrm{(б)}$

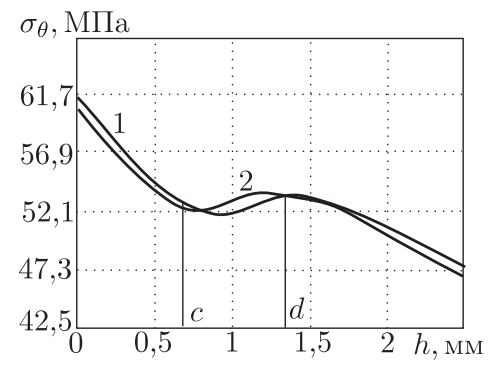

$a$

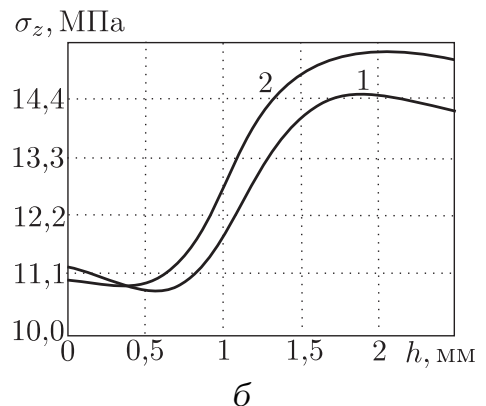

$б$

Рис. 3. Распределение окружных (а) и осевых (б) напряжений по толщине трубы $h=r-R_{1}$ на момент разрушения конструктивного элемента: 1 - локальная область; 2 - «холодная» область 
показаны на рис. 3 .

В зоне повышенных температур окружные напряжения (рис. 3, а, кривая 1) до участка активных химических превращений $c d$ имеют большее значение по сравнению с зоной пониженных температур (рис. 3, а, кривая 2). На участке $c d$ происходит их перераспределение и после этого участка восстанавливается первоначальное соотношение между напряжениями. Осевые напряжения (рис. 3, б) также претерпевают перераспределение в области активных химических превращений, но в результате локального прогрева их соотношение не становится исходным. Радиальные напряжения не испытывают заметного перераспределения как в области локального прогрева, так и в «холодной» области.

\section{БИБЛИОГРАФИЧЕСКИЙ СПИСОК}

1. Овчинников И.Г., Хвалъко Т.А. Работоспособность конструкций в условиях высокотемпературной водородной коррозии. Саратов: Сарат. гос. техн. ун-т, 2003. 176 с. [Ovchinnikov I. G., Khvalko T.A. Serviceability of structures under high-temperature hydrogen corrosion. Saratov: Sarat. Gos. Tekhn. Un-t, 2003. 176 pp.]

2. Арчаков Ю.И., Гребешкова И. Д. Водородная коррозия сталей в газовой фазе / В сб.: Коррозия и защита от коррозии (Итоги науки и техники). Т. 4. М.: Наука, 1975. C. 113-174. [Archakov Yu.I., Grebeshkova I. D. Hydrogen corrosion of steel in gas phase / In: Corrosion and Corrosion Protection (Results of Science and Technology). Vol. 4. Moscow: Nauka, 1975. Pp. 113-174].

3. Локощенко А. М. Длительная прочность металлов при сложном напряженном состоянии // Пробл. прочности, 1983. № 8. С. 55-59; англ. пер.: Lokoshchenko A. M. Stressrupture strength of metals in the complex stressed state// Strength of Materials. Vol.15, no. 8. Pp. 1098-1103.

4. Бубнов С.А. Учет водородной коррозии в расчете НДС толстостенной трубы в программном комплексе ANSYS / В сб.: Инновачионные технологии в обучении и производстве: Сб. научных трудов. Камышин, 2010. С. 23-26. [Bubnov S. A. Accounting of hydrogen corrosion in stress-strain state calculation of thick-walled tube in program complex ANSYS / In: Innovative technologies in teaching and production: Collection of scientific works. Kamyshin, 2010. Pp. 23-26].

5. Овчинников И. Г., Бубнов С.А. Применение программного комплекса ANSYS к pacчету толстостенного трубопровода, подвергающегося высокотемпературной локальной водородной коррозии // Изв. Сарат. ун-та. Нов. сер. Сер. Математика. Механика. Информатика, 2011. T. 11, №3(2). C. 100-102. [Ovchinnikov I. G., Bubnov S. A. Application of program complex ANSYS to calculation of the thick-walled pipeline which is exposed to high-temperature local hydrogen corrosion // Izv. Saratov. Univ. Mat. Mekh. Inform., 2011. Vol. 11, no. 3(2). Pp. 100-102].

6. Бубнов С. А., Овчинников И. Г. Моделирование ползучести и разрушения неравномерно прогретого толстостенного трубопровода в условиях высокотемпературной водородной коррозии // Вестн. Сам. гос. техн. ун-та. Сер. Физ.-мат. науки, 2011. №4(25). С. 6774. [Bubnov S.A., Ovchinnikov I. G. Simulation of creep and fracture of thick-walled nonuniformly heated pipeline under high-temperature hydrogen corrosion // Vestn. Samar. Gos. Tekhn. Univ. Ser. Fiz.-Mat. Nauki, 2011. no. 4(25). Pp. 67-74].

7. Бубнов А.А., Бубнов С. А., Овчинников И. И. Моделирование напряженного состояния и разрушения толстостенных трубопроводов в условиях водородной коррозии и неоднородного теплового поля. М.: Горячая линия - Телеком, 2011. 135 с. [Bubnov A. A., Bubnov S. A., Obchinnikov I. I. Modelling of stress state and fracture of thick-walled piping in hydrogen corrosion and heterogeneous thermal field. Moscow: Goryachaya Liniya - Telekom, 2011. 135 pp.] 
MSC: 74R20; 74S05

\title{
INVESTIGATION OF DESTRUCTION AND DECARBURIZATION KINETICS OF A THICK-WALLED TUBES UNDER HYDROGEN CORROSION
}

\author{
S. A. Bubnov ${ }^{1}$, I. I. Ovchinnikov ${ }^{2}$, A. A. Bubnov ${ }^{3}$
}

1 Balashov Institute (Branch) of the Saratov State University named after N. G. Chernyshevsky, 29, Karla Marksa st., Balashov, Saratov region, 412300, Russia.

2 Saratov State Technical University named after Yu. A. Gagarin, 77, Politekhnicheskaya st., Saratov, 410054, Russia.

3 Ryazan State Radio Engineering University, 59/1, Gagarin st., Ryazan, 390005, Russia.

E-mails: serbubnov@rambler.ru, bridgesar@sstu.ru

The behavior of the thick-walled pipeline under hydrogen corrosion is studied. The model example of thick-walled tube made of St. 20 steel with a region of local heating is considered. The internal and external pressures of hydrogenous medium are used as loading factors in the model example. The greatest effect of the value of internal pressure of hydrogenous medium on time to fracture is noted. Also, the faster decarburization in the region of high temperature is pointed out.

Key words: hydrogen corrosion, destruction, front of decarburization, thick-walled pipeline.

Original article submitted $02 / \mathrm{V} / 2011$;

revision submitted $06 / \mathrm{V} / 2012$.

Sergey A. Bubnov, Assistant, Dept. of Applied Informatics. Igor' G. Ovchinnikov (Dr. Sci. (Techn.)), Associate Professor, Dept. of Bridges and Transport Constructions. Alexey A. Bubnov (Ph. D. (Phys. \& Math.)), Associate Professor, Dept. of Computational and Applied Mathematics. 